\title{
The Use of Manufacturing Commands and Features in a CAD Interface to Familiarize Engineering Students with Manufacturing Equipment and Processes Capabilities
}

\author{
Mario H. Castro-Cedeno \\ Rochester Institute of Technology \\ 1 Lomb Memorial Drive \\ Rochester, NY 14623-5603
}

\begin{abstract}
Manufacturing features and commands such as cut, drill, mill, turn, etc. can be added to the interface of computer aided CAD software. The commands can be used to design simple to medium complexity parts by using commands in a way that resemble the creation of a manufacturing process plan. Students using the software can be introduced to the capabilities and relative advantages and disadvantages of manufacturing processes and equipment. The software can be used as an augmentation of conventional shop exercises where the students have to plan the steps needed to fabricate a part.
\end{abstract}

This research describes some of the previous research on design and manufacturing features and provides examples of how manufacturing commands have been added to Solidworks CAD software.

\section{Introduction}

The goal of seamlessly transferring information between design and manufacturing has received much research effort during the last 20 years and researchers have achieved significant success [1]. The high level of interest is due to the fact that large labor and time savings would be possible if a manufacturing process plan could be generated from the CAD model with no or minimal human intervention. Although much of the recent work is focused on converting design features into manufacturing features through feature recognition and translation [2], early researchers explored the possibility of driving CAD geometry creation directly with process plan compatible commands [3].

Modern, feature-based CAD software can be modified to include a language similar to the operations used on a process plan. The requirements are that 1) the design features of the CAD software can be translated into process plan actions and 2) that the CAD software must have a language that can be used to extend its capabilities. This research has focused on developing an interface for Solidworks CAD software to demonstrate the use of process planning and manufacturing commands that can be used to create simple parts. It is envisioned that students can use the modified software to learn what steps can be used to create a part. Most importantly, students can explore alternate approaches to making a part, and compare their advantages and disadvantages. 
The software can give students "shop" experience without having to use the equipment. This translates into savings in capital investment and the ability to train more students with limited equipment resources. For non-manufacturing majors, the software offers the opportunity of studying manufacturing equipment and process capabilities in depth, without worrying about the safe use of the equipment and other shop safety concerns.

\section{The Language of Manufacturing}

Manufacturing uses additive or protrusion processes such as welding or joining, and material removal or depression processes such as machining [4]. A third situation is plastic forming where material is moved but the volume remains constant. It is also important to note that manufacturing starts with the selection of material in "stock" or standard shapes. Table 1 lists some of the activities or actions that can be used to manufacture parts and can be used as commands in our manufacturing language. Italics are used for commands not presently implemented but planned for the near future. Note also that commands are grouped into three sections: 1) Stock Material, 2) Manufacturing and 3) Finishing.

Table 1 - Manufacturing Commands

Stock Material

Bar (round, rectangular, square)

Block

Casting

Pipe

Plate

Sheet

Tube
Manufacturing

Cut

Drill (thru, counterbore, countersunk)

Mill (face, end, horizontal)

Extrude

Turn

Join/Weld
Finishing

Paint

Plate

Polish

\section{Examples}

Solidworks and other modern CAD software can easily create all the stock material options on Table 1. For castings, 3D solids can be imported. The process of creating the solids can limit the options to the commercial sizes available. For Solidworks, the Application Programming Interface (API) allows the automation of the geometry creation. The user is asked to input the appropriate size dimensions. The stock material options are listed on a menu.

After the stock material geometry is created, the software introduces a new menu with the manufacturing options. Most of the manufacturing operations are available in the Solidworks software and can be re-named to match the manufacturing language. One example is the "Extrude-Cut" command which is used for "Cut". Occasionally, the manufacturing feature requires a combination of design features or commands and must be programmed as a Macrostatement. The milling operations, for example, can be programmed as "Cut-Sweep" commands and require the cross-section of the cutter tool and a path as input. A final example of a manufacturing command is the Join/Weld operation, which uses the Solidworks "Mate" command. 
Figures $1 \mathrm{a}$ and $1 \mathrm{~b}$ show the milling of a simple shape from a plate. Figure 1a show the plate with the path and cross-section requested as input and Figure 1b show the effect of the cut operation.

A simple application of the language is the creation of the simple bracket in Figure 2. It can be machined from a block or from welded plates that are machined to final dimensions after the welding operation. When the weldment is converted from an assembly into a part, additional operations such as milling, drilling of holes, etc. can be added.

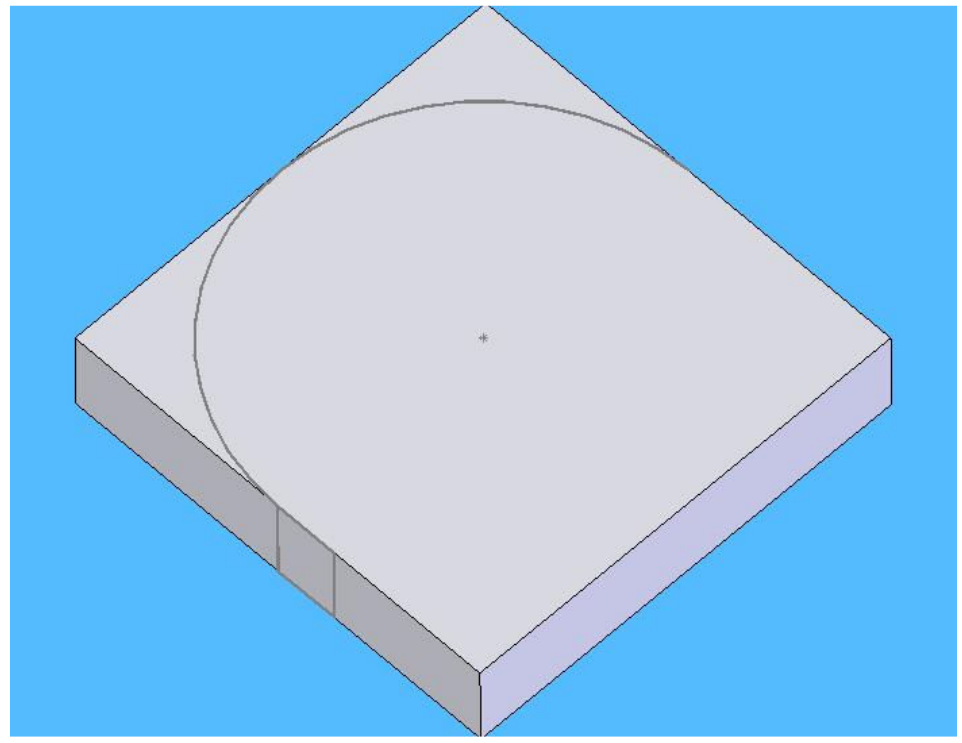

Figure 1a - Plate Showing Path and Cutter Cross-Section Input

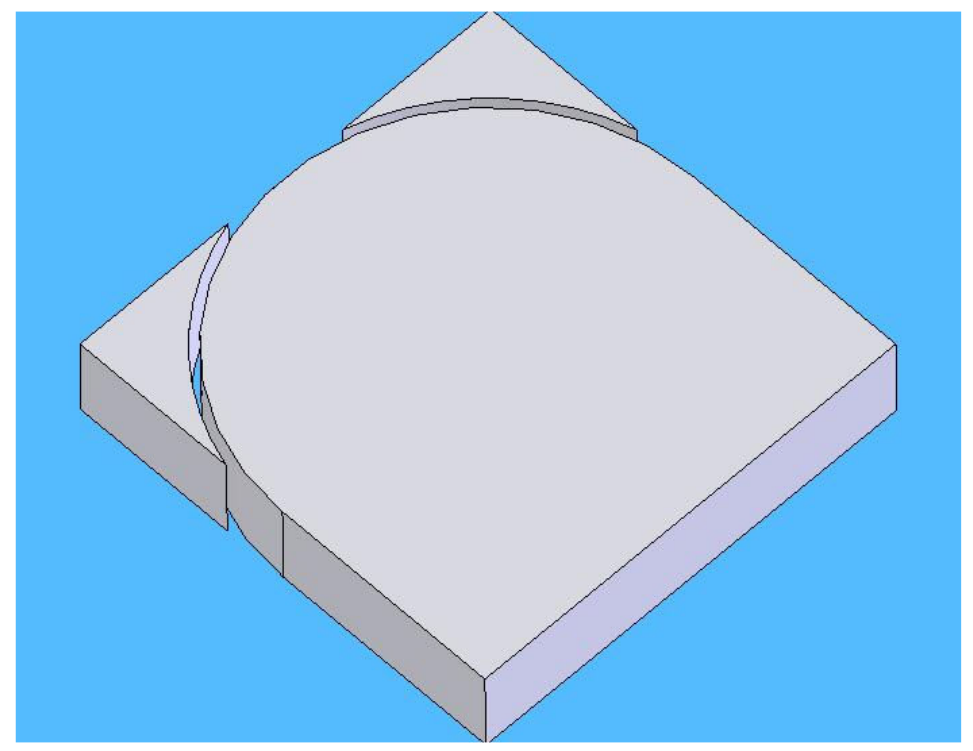

Figure $1 \mathrm{~b}-$ Cut Plate

Proceedings of the 2005 American Society for Engineering Education Annual Conference \& Exposition Copyright (C) 2005, American Society for Engineering Education 


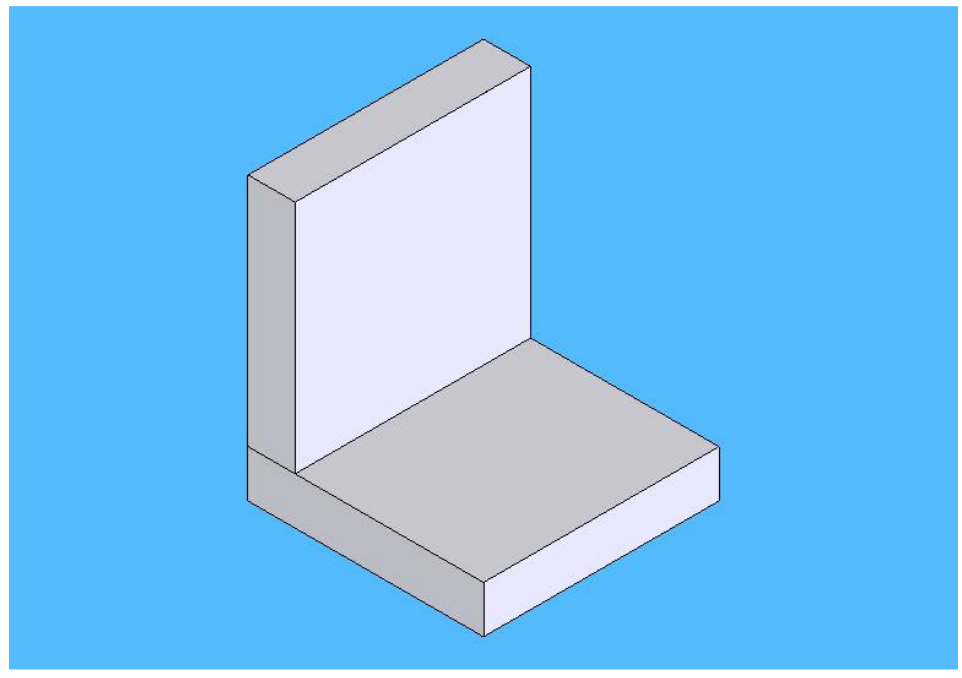

Figure 2 - Simple Bracket

\section{Future Extensions}

In the near future the software will be tested by users to increase user friendliness and reliability. Future plans include adding the commands for surface finish, a cost module so that the cost of making a part using different processes can be compared and a module to provide expert advice that will also constrain or limit commands not appropriate or recommended for the situation.

\section{Conclusions}

1. Manufacturing commands and features can be added to modern CAD software. These commands can be used to build CAD models of simple and medium complexity parts.

2. Students using the software can explore how finished parts can be made from the input material available in "commercial or stock" sizes. Students can also explore the use of manufacturing equipment and compare the relative advantages and disadvantages of different approaches to make a part.

3. Manufacturing students can use the software as a complement to conventional shop exercises and can gain shop experience in excess of the actual shop hours they are required and/or allowed to have.

4. Students in majors other than manufacturing can get a more complete and in-depth feel and understanding for manufacturing processes and equipment, and their limitations than they can get from lectures or demonstrations. Further, they do not have to worry about their personal safety and do not need expensive manufacturing equipment and facilities. 


\section{References}

[1] Han, JungHyun, Pratt, M. and Regli, W.C., "Manufacturing Feature Recognition from Solid Models: A Status Report" in IEEE Transactions on Robotics and Automation, Vol. 16, No. 6, December 2000, p. 782.

[2] Li, Wei Dong, Ong. S.K. and Nee, A.Y.C., "Recognizing Manufacturing Features from a Design-by-Features Model" in CAD Computer Aided Design, Vol 34, No. 11, Sept 15 2002, p. 849.

[3] Owusu-Ofori, S.P., "Part Design Using Manufacturing Features" in Journal of Intelligent Manufacturing, Vol. 5, No. 1, February 1994, p. 55.

[4] Chamberlain, M.A., Joneja, A. and Chang, T-C, "Protrusion-Features Handling in Design and Manufacturing Planning" in Computer Aided Design, Vol. 25, No 1, Jan 1993, p. 19.

\section{Mario H. Castro-Cedeno}

Mario H. Castro-Cedeno is an Assistant Professor in the Department of Mechanical and Manufacturing Engineering Technology and Packaging Science at the Rochester Institute of Technology (RIT) in Rochester, NY. He teaches courses in introduction to manufacturing, materials technology and computer aided design to engineering technology undergraduates. Before joining RIT in December of 2003 he accumulated 30 years of engineering and management experience at various firms, including NASA and General Electric.

Mr. Castro-Cedeno was born in Puerto Rico and obtained his B.S. and M.S. in Mechanical Engineering from the University of Puerto Rico and a Master of Engineering degree in Materials Science and Engineering from the University of California at Berkeley.

Proceedings of the 2005 American Society for Engineering Education Annual Conference \& Exposition Copyright (C) 2005, American Society for Engineering Education 